\title{
McLuhan y el método para el estudio de los medios: El enfoque estructural
}

\author{
Alberto J. L. Carrillo Canán
}

Benémerita Universidad Autónoma de Puebla

\begin{abstract}
Para el estudioso de los medios, cada detalle del mosaico del mundo contemporáneo está cargado de vida significante.
\end{abstract}

MCLUHAN

\section{Resumen}

En este texto abordaremos el método de McLuhan principalmente a partir de algunas observaciones muy reveladoras en su obra Understanding Media. The Extensions of Man (1964). Proponemos como un concepto básico para entender el método de McLuhan, el de estructura o, bien, "aproximación estructural" (McLuhan 1964, p. 13) a los medios, un enfoque que McLuhan distingue de forma explícita de la "aproximación convencional" (p. 71), que sería el de los tratados sistemáticos que siguen un desarrollo lógico a partir de ciertos presupuestos. McLuhan (1964) mismo utiliza con frecuencia el término "estructura" para referirse a su objeto de estudio, es decir, los medios, al hablarnos incluso del "estudioso de las estructuras mediáticas" (p. 195) y, según veremos, en McLuhan existe una correlación o paralelismo entre los medios entendidos como "estructura" y el método o "aproximación estructural” para su estudio. El método correspondería, pues, a su objeto o tema.

Palabras Clave: McLuhan, medios, aproximación estructural, Understanding Media. The Extensions of Man.

\section{Abstract}

In this text we will approach McLuhan's method mainly from some very revealing observations in his work Understanding Media. The Extensions of Man (1964). We propose as a basic concept to understand McLuhan's method, that of structure or "structural approach" to the media, an approach that McLuhan explicitly distinguishes from the 
"conventional approach", which would be the systematic treaties that follow a logical development from certain presuppositions. McLuhan (1964) himself frequently uses the term "structure" to refer to his object of study, that is, the media, even talking about the "scholar of media structures" (p. 195) and, as we shall see, in McLuhan there is a correlation or parallelism between the media understood as "structure" and the method or "structural approach" for its study. The method would correspond, then, to its object or theme.

KEYWORDS: McLuhan, media, structural approach, Understanding Media. The Extensions of Man.

La teoría mediática de Marshall McLuhan queda expuesta en una serie de libros —algunos editados de manera póstuma por algún colaborador - en los que aparecen numerosos señalamientos acerca del método que este autor aplica en sus estudios mediáticos. Sin embargo, tales señalamientos acerca del método tienen la misma característica general de su obra, consistente en no ser sistemáticos de una manera reconocible, McLuhan diría "lineal" o "secuencial", de acuerdo con la forma de la experiencia propia de lo que McLuhan mismo llama el hombre occidental o "alfabetizado". De hecho, nada de lo que McLuhan expone, incluidas sus observaciones sobre la manera o método para el estudio de los medios, tiene el carácter sistemático de un tratado académico. En este texto abordaremos el método de McLuhan principalmente a partir de algunas observaciones muy reveladoras en su obra Understanding Media. The Extensions of Man (1964). Proponemos como un concepto básico para entender el método de McLuhan, el de estructura o, bien, "aproximación estructural' (McLuhan 1964, p. 13) a los medios, un enfoque que McLuhan distingue de forma explícita del "aproximación convencional" (p. 71), que sería el de los tratados sistemáticos que siguen un desarrollo lógico a partir de ciertos presupuestos. McLuhan (1964) mismo utiliza con frecuencia el término "estructura" para referirse a su objeto de estudio, es decir, los medios, al hablarnos incluso del "estudioso de las estructuras mediáticas” (p. 195) y, según veremos, en McLuhan existe una correlación o paralelismo entre los medios entendidos como "estructura" y el método o "aproximación estructural" para su estudio. El método correspondería, pues, a su objeto o tema. 
Una vez clarificado el método estructural de McLuhan, su manera general de exponer resulta comprensible, pierde el aspecto de un simple conjunto de observaciones casuales y hasta cierto punto repetitivas $y$, entonces, sirve como un método a aplicar de manera razonable por productiva y clarificadora, a los fenómenos de nuestro entorno mediático contemporáneo — de hecho, de cualquier entorno mediático-.

\section{La realidad estructural del medio y su "captación" no discursiva}

El texto Understanding Media en su conjunto insiste en la idea de que para las personas alfabetizadas el estudio de los medios, de acuerdo con lo que se debe considerar la "naturaleza del medio" (McLuhan 1964, p. 11), es algo "muy difícil" (McLuhan 1964, p. 164), de hecho, McLuhan considera que tal estudio es imposible sin un cambio radical del modo de pensar fomentado por la sociedad alfabetizada. El tema de los efectos sociales y mentales del alfabeto es crucial en la obra de McLuhan, inclusive, por supuesto, el problema del método. Por lo pronto, baste con decir que según McLuhan (1964) "(...) la causa oculta de nuestro sesgo occidental hacia la secuencia como 'lógica' [reside] en la tecnología omnipresente del alfabeto (...)" (p. 85), tecnología que entrena para hábitos que suponen "la homogeneidad, la uniformidad y la continuidad" (p. 87). ${ }^{1}$ Tales supuestos están en la base de la organización conceptual de la experiencia en secuencias de razonamientos con algún tipo de lógica. ${ }^{2}$ Por ello, según McLuhan (1964) es la escritura alfabética la que generó el "condicionamiento psicológico" (p. 136) necesario para pensar de manera secuencial, es decir, para hacer razonamientos lógicos de todo tipo. Son justo estos hábitos mentales los que dificultarían al hombre alfabetizado entender los medios, porque los medios tienen, según lo propone McLuhan, un carácter estructural.

Para discutir la problemática recién señalada, examinaremos un pasaje clave del texto Understanding Media, en el que procederemos por pasos:

\footnotetext{
Sobre esto $c f r$. Carrillo 2017, pp. 1-27.

2 Sobre esto $c f r$. Carrillo 2004, pp. 1-5.
} 
Hoy en día los estudiosos tienen una conciencia muy clara de una discrepancia entre las maneras en las que tratan sus temas y el tema mismo. Los estudiosos de las escrituras, tanto del viejo como del nuevo testamento, dicen frecuentemente que mientras que su tratamiento tiene que ser lineal, el tema no lo es. El tema remite a las relaciones entre Dios y el hombre, y entre Dios y el mundo, y a la relación entre el hombre y su vecino; todos subsisten juntos, y actúan y reaccionan el uno sobre el otro al mismo tiempo (McLuhan 1964 , p. 25$).^{3}$

La noción de simultaneidad — como estructura de la experienciaes algo que McLuhan opone sistemáticamente a la secuencialidad, como quedará claro, pero por lo pronto notemos que lo que McLuhan describe en el pasaje en cuestión es una estructura, es decir, una entidad que consta de elementos que tienen su sentido de manera relacional o, dicho de otra manera, de manera relativa los unos a los otros: Dios, el hombre, el mundo, el prójimo. El sentido que pueda tener cada uno de estos elementos en sí mismo queda supeditado al sentido que tienen en su conjunto de relaciones. No son elementos absolutos sino relativos. El ejemplo de McLuhan es dinámico, sin embargo, la idea se puede entender muy claramente con un ejemplo no dinámico.

Supongamos tres puntos, A, B, C, en un plano, que forman un triángulo. Se trata no sólo de puntos en sí mismos sino de puntos que tienen el sentido de formar un triángulo. Supongamos que agregamos un cuarto punto, $\mathrm{D}$, que genera un rombo, esto significa que sin más el sentido de los puntos A, B y C cambia de manera radical. Por ejemplo, el punto A sigue siendo el mismo, tiene exactamente la misma ubicación en el espacio, sea visto como punto del triángulo o como punto del rombo, en ambos casos es el mismo punto y, en sí mismo, aislado, tiene el mismo sentido, a saber el de ser un punto diferente de cualquier otro en su ubicación singular, sin embargo su sentido estructural es totalmente diferente en cada caso. En el primer caso A es un punto de un triángulo, en el segundo caso ese mismo punto es el punto de un rombo, lo cual es algo por completo distin-

3 Las cursivas en una cita son nuestras siempre, a menos que se indique otra cosa. 
to. Como entidad geométrica independiente, absoluta, no relativa, A tiene, digámoslo así, la misma realidad o sentido substancial, pero ese mismo punto, invariable como substancia, convertido en elemento estructural, es decir, como nodo de una estructura, tiene dos sentidos totalmente diferentes, al lado, pero independientes de su sentido substancial. Uno es el punto en sí mismo y otro es ese mismo punto como vértice de un triángulo y otro más es dicho punto como vértice de un rombo. Estos dos son sentidos no absolutos sino relativos a otros elementos.

Sin entrar en mayores complicaciones digamos que un elemento de un conjunto es un elemento estructural si adquiere su sentido a partir de las relaciones con los otros elementos de dicho conjunto. ${ }^{4}$ El conjunto, entonces, ya no es un conjunto, una simple colección de elementos aislados y autónomos, absolutos, sino una estructura, y sus elementos son, justamente, elementos estructurales, con sentido relativo de los unos a los otros, es decir a la estructura como tal. En el texto citado McLuhan (1964) nos da una versión dinámica de esto al decirnos que el tema de los eruditos bíblicos “(...) remite a las relaciones entre Dios y el hombre, y entre Dios y el mundo, y a la relación entre el hombre y su vecino; todos subsisten juntos, y actúan y reaccionan el uno sobre el otro al mismo tiempo." (pp. 25-26) Sin embargo, dinámica o estática, una estructura es una estructura.

Continuemos con el examen del pasaje. Sin solución de continuidad McLuhan (1964) dice que

[e]l modo de pensar hebreo y oriental aborda el problema y su resolución desde el inicio de la discusión, de una manera típica de las sociedades orales [prealfabéticas] en general. El mensaje entero se traza y retraza, entonces, una y otra vez, sobre las vueltas de una espiral concéntrica, con una redundancia aparente. Uno puede parar en cualquier momento después de unas pocas oraciones y tener el mensaje completo, si es que uno está preparado para "desenterrarlo" (p. 26).

4 Nótese que si se toma el conjunto de relaciones entre los elementos de la estructura, lo que equivale a la estructura misma, entonces hay una simultaneidad de los elementos. La simultaneidad de los elementos es el carácter temporal de una estructura. 
De especial importancia en la cita es la noción de una "redundancia aparente" en las "vueltas" sobre una "espiral". Se trata de una analogía. Para aclarar la idea tenemos como ejemplo el tratamiento que McLuhan hace de cualquier medio en el libro que nos ocupa, Understanding Media, por ejemplo, la TV.

A pesar de que en el texto hay un capítulo dedicado a la TV, McLuhan se refiere a ella múltiples veces tanto antes como después de dicho capítulo, incluso expresa las mismas ideas — "trazando y retrazando el mismo mensaje" - , lo que sugiere una redundancia, simples "vueltas" o retornos; sin embargo, en cada uno de esos tratamientos o menciones la TV es considerada en relación con medios diferentes, digamos, el periódico, la caricatura, el cine, ciertos deportes, etcétera. De tal manera que en cada "vuelta" la naturaleza mediática de la TV va quedando cada vez más clara, justamente al verla en su relación con dichos otros medios. Entonces, aunque se vuelve una y otra vez a la TV, lo que se comprende de ella puede ser incluso lo mismo pero con mayor profundidad o riqueza, es decir, la comprensión o conciencia de lo que es la TV cada vez que ésta es abordada, ha crecido, se está en una parte superior de la "espiral", en el "mismo punto" de la "vuelta", pero a una altura mayor. Por eso la redundancia es solamente aparente. En otras palabras, McLuhan rechaza el tratamiento que consistiría en un examen secuencial de las características de la TV en sí misma, una por una. Un análisis de tal tipo, algo así como una teoría estándar de la TV, sería, según McLuhan, totalmente inapropiado para entender su naturaleza mediática — que no la tecnológica propiamente dicha—. En términos de teoría mediática un estudio de la TV en sí misma es imposible. McLuhan (1964) insiste en que se tiene que tratar con "la situación ambiental total" (p. 11), es decir con el entorno mediático o la "matriz [mediática] dentro de la cual opera cada medio” (p. 11). En este contexto se entiende la insistencia de McLuhan (1964) en utilizar expresiones como "el mundo de la estructura y la configuración" (p. 13), "el patrón total (...) como unidad" (p. 13), o bien la "idea integral de estructura y configuración” (p. 13) y también la "interacción de planos" (p. 13). Nótese que, de hecho, otros términos mcluhanianos para hablar de estructura son "configuración" y "patrón".

Por lo pronto, resulta comprensible que McLuhan (1964) "[e]n vez de trabajar con problemas 'especializados”, utilice lo que él llama la "aproximación estructural" (p. 13) a los medios y que se interese en 
los "componentes y propiedades estructurales" (p. 49) de los medios, en la "vida de las formas y las estructuras" (p. 65). Lo cierto es que McLuhan (1964) opone la "aproximación estructural" a la "aproximación convencional" a los medios (p. 71), determinada, ésta, por la "estructuración lineal de la vida racional mediante la alfabetización fonética” (p. 85). Continuemos con nuestro examen del pasaje de interés.

Un poco más adelante, McLuhan (1964) se refiere a la "forma redundante" y nos dice que

(...) lo concéntrico, con su intersección infinita de planos, es necesario para la captación [insight]. De hecho, ello mismo es la técnica de la captación, y como tal es necesaria para el estudio de los medios, puesto que ningún medio tiene su significado o existencia solitario, sino solamente en la interacción constante con otros medios (p. 26).

Respecto de esta cita empecemos por señalar que la noción de insight, si bien tiene el sentido de entendimiento o comprensión, indica un cierto tipo de los mismos, a saber, intuitivo ${ }^{5}$, lo cual, es opuesto a lo discursivo o lineal. ${ }^{6}$ No se trata de que se entienda a través de una explicación, parte por parte, es decir, de manera secuencial, sino que aun habiendo ésta, de pronto, "de un golpe" — como gustaban decir los fenomenólogos como Husserl ${ }^{7}$ — se capta o entiende algo.

5 Ver por ejemplo la definición de la palabra insight en el diccionario Merriam-Webster en línea.

6 Sobre esto ver Kant (Kritik der reinen Vernunft, 1787): "Wir haben in dem angeführten Beispiele nur deutlich zu machen gesucht, welcher große Unterschied zwischen dem diskursiven Vernunftgebrauch nach Begriffen und dem intuitiven durch die Konstruktion der Begriffe anzutreffen sei." Traducción: "En los ejemplos presentados hemos tratado de poner en claro la gran diferencia que se encuentra entre el uso discursivo de la razón según conceptos y el intuitivo a través de la construcción de los conceptos." (Capítulo 131 en la navegación de la página WEB de referencia).

7 Esta idea se encuentra, por ejemplo, en la distinción que hacer Husserl entre los "actos intuitivos" y los "actos categoriales". La "intuición", que siempre tiene una estructura análoga a la de la percepción sensorial, presenta sus objetos de manera "simple", "directa", "de un golpe [mit einem Schlag]" LI 788 (Lolgische Untersuchungen, HUA XIX 676). 
Entonces, aquí encontramos la idea de la oposición entre la "aproximación tradicional" — "lineal" — y el "estructural”, que ahora se nos revela como, por lo menos, con un momento intuitivo decisivo. Esta oposición entre lo intuitivo y lo discursivo — aunque McLuhan la tematice bajo otros términos como simultáneo y secuencial, por ejemplo- es la razón de que McLuhan no utilice prácticamente la palabra consciousness sino la palabra awareness, y a saber, en el sentido de una captación simultánea de múltiples elementos, es decir, de captación de una situación, la cual siempre es una estructura en el sentido discutido arriba. En este sentido la referencia de McLuhan (1964) a un "(...) tipo de captación [awareness] total, configuracional [estructural], que revela por qué el medio es socialmente lo que importa [the medium is socially the message $]^{8}$ (...)" (p. 10), es una referencia crucial y clarificadora. ${ }^{9}$

El segundo asunto a resaltar es la idea final de lo citado en el sentido de que "(...) ningún medio tiene su significado o existencia solitario, sino solamente en la interacción constante con otros medios". Con esto último queda claro que McLuhan en realidad piensa una "aproximación estructural" a los medios, a todos y cada uno. Es claro que un medio, digamos una computadora, tiene una realidad substancial, que es la que sería el objeto de un estudio técnico y también por parte de las ciencias naturales. Así, independientemente de para qué y cómo se utilice, la computadora tiene ciertas características técnicas y naturales, justamente, substanciales, como los materiales de que está hecha, sus dimensiones, su forma objetiva, sus capacidades de memoria, su conectividad, su consumo de energía, etcétera. Éstas, las propiedades objetivas y tecnológicas de la computadora, son el portador de sus propiedades estructurales pero en su totalidad diferentes de éstas, de manera análoga a como en el

8 Adelante nos referiremos a la idea de que the medium is the message, así como a su traducción correcta: el medio es lo importante, lo que importa, lo que cuenta.

9 El asunto de la awareness como captación o conciencia de una configuración, situación o estructura, como captación de elementos en simultaneidad, se repite por todo el libro, y el siguiente fragmento también es muy instructivo al respecto: "Con la velocidad instantánea las causas de las cosas comienzan a emerger de nuevo en la conciencia [awareness] como no lo habían hecho con las cosas en secuencia (...)" (McLuhan, 1964, p. 12). El asunto de la velocidad es crucial y será tratado abajo. 
ejemplo geométrico el punto A en sí mismo, substancialmente, es el portador de su sentido estructural como punto de un triángulo, en un caso, y como punto de un rombo, en el otro caso.

A fin de cuentas, la realidad estructural del medio impone su captación estructural, captación que es muy diferente de los hábitos discursivos con su procedimiento de análisis y de secuencia lógica.

\section{El método visto inmanentemente}

El pasaje que examinamos tiene una continuación importante que plantea dos problemas. En este apartado nos concentraremos en el primero de estos dos problemas y es el del origen del método de McLuhan, visto como origen inmanente, es decir, interno al tema que es el objeto del método, a saber, a los medios mismos. Para explicar la inmanencia del método, su origen inmanente al tema, empecemos por citar la continuación del pasaje examinado. McLuhan (1964) nos dice que:

[1]as noveles estructuración y configuración eléctricas de la vida chocan más y más con los viejos procedimientos y herramientas de análisis lineales y fragmentarios de la época mecánica. Más y más nos movemos del contenido de los mensajes al estudio del efecto total (p. 26).

Esta cita es uno de múltiples pasajes en los que McLuhan (1964) apunta a que es el entorno mediático "eléctrico", el que, por sí mismo, genera nuevas condiciones de la conciencia, justamente en su versión de "captación [awareness] total instantánea" (p. 13) ${ }^{10}$. En otras palabras, McLuhan no se presenta a sí mismo como alguien que a partir de una genialidad personal gratuita haya llegado a algo

10 Recuérdese que ya señalamos la oposición entre simultaneidad y secuencialidad; nótese ahora que si la secuencialidad queda excluida lo que se tiene es la instantaneidad: la "captación instantánea del todo" (McLuhan, 1964, p. 12). En adición nótese que si la intuición es lo opuesto al discurso, es decir al recorrido secuencial, entonces intuición significa captación "de un golpe", como en la ya señalada tradición fenomenológica. Ver la nota de pie de página 65. 
nuevo por completo; más bien sería un entorno mediático específico lo que habría hecho posible a McLuhan mismo ${ }^{11}$ - y a otros teóricos que el propio McLuhan señala a lo largo de sus textos, incluido Understanding Media, que hacen aportaciones múltiples para la comprensión de los medios-. Lo importante es, pues, que sería la "época eléctrica" (McLuhan, 1964, p. 5) misma lo que posibilitaría el método de McLuhan. El método no sería algo paralelo o añadido a la realidad mediática desde una esfera extraña a ella o independiente, sino un momento de conciencia interno, inmanente a la misma, en tanto entorno mediático "eléctrico", de hecho, el método sería uno de los efectos de dicho entorno, parte de su "efecto total". Antes de continuar con el pasaje clave que nos interesa respecto del método, aún debemos aclarar la idea de la relación entre el método y el entorno o "época eléctrica". Éste es el segundo problema que teníamos en mente al inicio de esta sección de nuestro texto.

\section{El método y la "época eléctrica"}

Los medios "eléctricos" - el telégrafo, el radio, la TV, el satélite, el avión, la misma luz eléctrica, entre otros - son definidos por McLuhan como aquellos que anulan el espacio y el tiempo, su efecto, es decir, su "mensaje", es la "implosión", para empezar en tanto anulación de las distancias temporales y espaciales, al convertir al mundo en una "aldea". McLuhan (1964) lo formula de la siguiente manera:

Después de tres mil años de explosión especialista y de especialismo y alienación incrementados (...) nuestro mundo ha devenido compresivo en una inversión dramática. En tanto contraído eléctricamente, el globo ya no es más que una aldea. La velocidad eléc-

11 En este sentido, McLuhan está en una tradición compartida por otros pensadores "históricos", como Hegel y Marx quienes, por mucho que aprecien sus propias aportaciones intelectuales, ven en un cambio de situación histórica la condición de posibilidad de pensar lo que ellos piensan. Tal vez ellos mismos se verán como geniales, pero postulan que su genialidad tiene el marco para su realización en una situación que los transciende o los engloba, posibilitándolos. 
trica, al acercar entre ellas todas las funciones sociales y políticas en una implosión súbita, ha incrementado la conciencia humana (p. 5).

La anulación del tiempo y el espacio significa en primer lugar que las "funciones sociales y políticas" dejan de estar ligadas a tiempos y lugares específicos, "especializados". No podemos hacer una discusión de esto aquí, pero una idea de ello la tenemos en lo que ahora llamamos dispositivos móviles y el caso del teléfono inteligente es paradigmático; no sólo no está en un lugar definido sino que es móvil, además él mismo es una implosión de funciones, dado que reúne un número creciente de aplicaciones que, en particular, hacen que la cámara fotográfica, la de video, el escáner, la grabadora de sonido, la linterna, la brújula, el giroscopio, el velocímetro, el altímetro, etcétera, etcétera, y el teléfono mismo, dejen de ser aparatos independientes, especializados. Más aún, jugar, comunicarse, trabajar de diferentes maneras, monitorear la propia salud, el ejercicio, las ubicaciones, etcétera, son otras funciones que no son más que modos de uso del dispositivo móvil, y en la medida en la que esté uno conectado a la WEB, el tiempo y el lugar son irrelevantes para realizar las funciones y para utilizar las posibilidades antedichas. El dispositivo móvil comprime, por así decirlo, el aquí y el ahora son irrelevantes, todas esas funciones se actualizan o ejecutan deslocalizadas temporal y espacialmente. Todo lo señalado ha implosionado, es decir, se ha concentrado en el dispositivo móvil, que por definición no está ligado ni a tiempo ni a lugar algunos. Así pues, el dispositivo móvil es emblemático del fenómeno de la "implosión" al que se refiere McLuhan, a pesar de que él nunca lo experimentó.

El dispositivo móvil muestra la irrelevancia del recorrido secuencial de lugares y de la secuencia temporal correspondiente para realizar de forma separada y especializada funciones humanas, pero la implosión no está sólo en la anulación de las viejas secuencias espaciales y temporales que exigía el modo especialista de existencia - la "explosión" o fraccionamiento y desagregación-, sino que también está en la inmediatez de los efectos a partir de las causas, o de las reacciones a partir de las acciones, todo ocurre "instantáneamente". Oponiendo la "época eléctrica” a la "mecánica”, es decir, la de la "explosión", con su especialismo y su fragmentación, McLuhan (1964) nos dice que: 
[e]n la época mecánica, ahora en retroceso, se podía emprender muchas acciones sin gran preocupación. El movimiento lento aseguraba que las reacciones se demoraran periodos de tiempo considerables. Hoy en día la acción y la reacción ocurren casi al mismo tiempo (p. 4).

En otras palabras, la velocidad de la luz, velocidad que en términos prácticos dados por el tamaño de la Tierra, hace nugatorio el espacio, es decir, la distancia, como factor que separa la acción de la reacción, del efecto, también hace que el tiempo deje de tener importancia. Un ejemplo claro de esto fue el efecto que tuvo aquella famosa conversación privada de Trump en un vestidor, siete años antes de las elecciones presidenciales en Estados Unidos en noviembre de 2016. El escándalo suscitado por esa conversación fue como si acabara de pasar, más aún, como si los oyentes de la grabación hubieran sido testigos de la misma, es decir, no sólo los siete años, también el lugar privado de la conversación, un vestidor para caballeros, fue anulado; todo fue como si Trump hubiera hablado delante de cada persona que tuvo acceso a la grabación a través de los medios. Los políticos se cuentan hoy en día entre las personas más conscientes de que debido a los medios de comunicación "eléctricos" — como lo diría McLuhan - una acción puede tener efectos instantáneos desligados del tiempo y del espacio donde ella ocurre.

Por ahora lo que importa es la noción de instantaneidad del efecto, lo que está ligado con lo que hoy en día se expresa con la noción de "tiempo real”. Dado que el mundo está interconectado "eléctricamente", en tiempo real, en cualquier punto del globo hay acceso a la información total de manera simultánea, lo que ocurre en cualquier punto se conoce en todos lados y tiene efectos inmediatos — por ejemplo, políticos o financieros_- Esta simultaneidad de la información y de los efectos de los medios eléctricos hace imposible el análisis, es decir, el examen secuencial de la información, pieza por pieza, al tiempo que también excluye la existencia de efectos aislados, limitados a lugares especializados — de ahí la noción del "efecto total" en la última cita-. Con ello, la linealidad secuencial como forma de la conciencia proveniente del modelo alfabético de la lectura y la escritura - queda inhabilitada como modo de la 
orientación en el mundo. ${ }^{12}$ Ahora podemos regresar al pasaje sobre el método.

Apenas dos oraciones más delante de la mención del "efecto total”, McLuhan (1964) nos dice que "[e]l interés en el efecto, más que en el significado, es un cambio básico en nuestro tiempo eléctrico, dado que el efecto involucra la situación total y no un único nivel del movimiento de la información” (p. 26, c. a.). ${ }^{13}$ Como vemos en la cita, McLuhan (1964) contrapone el "efecto" al "significado" y, de hecho inmediatamente, se refiere al "reconocimiento de este asunto del efecto más que de la información” (p. 26). La "información” vale en este caso como el "significado" o "contenido" de un medio, cosa que es necesario examinar para entender la noción de McLuhan sobre el método para el estudio de los medios.

\section{El medio y su contenido}

McLuhan sostiene de diversas maneras y a lo largo de todo el libro Understanding Media que los hábitos alfabéticos de pensamiento, que se manifiestan en la "aproximación convencional" a los medios, son los que llevan a que se ponga atención al "contenido" de los medios, no a "lo que importa (the message)", es decir, no al medio mismo, porque es el medio, no su contenido, lo que tiene el "efecto total". Lo primero, entonces, es precisar la diferencia entre el "medio" y su "contenido". Esto se puede hacer con base en un pasaje central en el capítulo uno del texto que nos ocupa. El pasaje reza lo siguiente:

12 McLuhan (1964) opone "conciencia total instantánea” (p. 13), ya considerada arriba $-\mathrm{y}$ que corresponde a la conciencia intuitiva kantiana o conciencia "en un golpe" husserliana-, y que ahora quedaría claro que es característica de la "era eléctrica", al "punto de vista", el cual sería parcial, limitado a un aspecto, y sobre este nos dice que "[e]l carácter parcial y especializado del punto vista, por noble que sea, no sirve para nada en la época eléctrica” (p. 5).

13 La abreviatura c. a. significa que las cursivas son del autor del texto citado. Como en este caso ya McLuhan usó las cursivas nosotros enfatizamos al utilizar el subrayado. 
(...) "el medio es lo que importa" [the medium is the message $]^{14}$ porque es el medio el que configura y controla la escala y la forma de la asociación y la acción humana. El contenido o los usos de tales medios son tan variados como carentes de efecto [ineffectual] para configurar la forma de la asociación humana. De hecho, es típico que el "contenido" de cualquier medio nos ciega frente al carácter del medio (McLuhan, 1964, p. 9).

En primer lugar, sobresale la muy conocida tesis de McLuhan, que además es el título del primer capítulo del libro, "el medio es lo que importa", y, claro, importa frente a lo secundario, lo "carente de efecto", que es el "contenido o los usos de los medios". La complementaridad de ambas tesis es evidente: al tratarse de "la escala y la forma de la asociación humana", lo eficiente, lo que tiene efectos, es el medio mismo, mientras que su "contenido o sus usos" no tienen ningún efecto sobre ellas, son ineficientes a este respecto. Por eso "el medio es lo que importa", "lo que cuenta" (the message). Aunque McLuhan usa profusamente el término "contenido" — a veces "significado"-, en esta ocasión lo distinguió con claridad de los "usos". La razón es clara; los medios de comunicación en sentido estricto tienen "significados" o "mensajes" en el sentido convencional de mensajes discursivos, equivalentes a textos; comunican significados. Sin embargo, lo que no es un medio de comunicación en sentido estricto, digamos el ferrocarril o el aeroplano - estos dos son medios de transporte, no de comunicación-, la luz eléctrica o una máquina (McLuhan, 1964, p. 7), tienen "contenido" en el sentido de tener usos. Tales medios no comunican nada, no portan ningún significado discursivo, sino que simplemente tienen usos. En general McLuhan subsume tanto los significados discursivos de los medios que lo tienen, los medios de comunicación en sentido estricto, como los usos de los medios restantes, bajo el término "contenido", y la tesis importante es que el hombre alfabetizado se fija justo en el "contenido" de cada medio así entendido y desatiende el medio mismo, es decir sus efectos sobre "la escala y la forma de

14 La famosa frase de McLuhan, "the medium is the message" suele traducirse literal, pero de manera incorrecta, en términos semánticos, como "el medio es el mensaje", pero la traducción correcta es que "el medio es lo importante", "lo que cuenta", "lo que importa". 
la asociación humana”. Es el sesgo del hombre alfabético hacia el contenido del medio lo que hace que para dicho hombre “(...) sea una sacudida el que se le recuerde que, en términos operativos y prácticos, el medio es lo que importa (...)" (McLuhan, 1964, p. 7), que es como empieza el capítulo uno del libro.

La tercera tesis en el pasaje citado sobre el medio y su contenido o usos, es que éste o estos “(...) nos ciega[n] frente al carácter del medio". En otras palabras, por atender al contenido del medio —en el sentido amplio discutido en el párrafo anterior - desatendemos al medio en sus efectos sociales. La inefectualidad del contenido del medio es subrayada por McLuhan ya en los dos primeros párrafos del capítulo uno de Understandig Media. Demos, para empezar, el ejemplo de que "(...) mediante la aceleración de la tasa de transporte, el aeroplano tiende a disolver la forma ferroviaria de la ciudad, la política y la asociación, con total independencia de para qué se usa el aeroplano” (McLuhan, 1964, p. 8). A lo que McLuhan (1964) añade apenas unas palabras adelante que "(...) es irrelevante si la luz [eléctrica] se utiliza para una cirugía cerebral o para un juego nocturno de baseball (...)" (p. 8), porque lo que importa es que dicha luz reconfigura "la escala y la forma de la asociación y la acción humana" al hacerla independiente de la luz diurna. Nótese que tanto el aeroplano como la luz eléctrica se pueden usar para muchísimas acciones, pero lo importante de ello es la transformación de la forma de la existencia que empieza por el cambio de la forma de la experiencia, del set mental del hombre que dispone de dichos medios. Así, por ejemplo, en la actualidad ya ni el agricultor parte de que sólo puede laborar bajo la luz del sol. En condiciones "eléctricas" la gente ya no es como el campesino que se recogía a descansar como las gallinas, al ponerse el sol, y se disponía a laborar con el canto del gallo. Ahí donde hay luz eléctrica, con independencia de para qué se le utilice, el hombre ya no tiene una actitud gallinácea frente a la vigilia y al sueño.

\section{La forma secuencial}

Un ejemplo adicional y muy conveniente de la inefectualidad del "contenido" del medio respecto de la "forma social" es el que se refiere a la máquina. McLuhan (1964) nos dice que: 
[e]n términos de las maneras en las que la máquina alteró las relaciones de los unos con los otros y respecto de nosotros mismos, no importa ni en lo más mínimo si ella arrojaba hojuelas de maíz o cadillacs. La reestructuración del trabajo y la asociación humana fue configurada por la técnica de fragmentación, que es la esencia de la tecnología maquinista (p. 7).

Dicho de otra manera, lo importante de la tecnología maquinista es que continúa la restructuración de la psique y de la vida en su conjunto, que empezó con la escritura alfabética en tanto primera "técnica de fragmentación"; por lo demás, la relación entre la escritura alfabética y la máquina se consolidaría con la imprenta. Según McLuhan (1964),

[1]a imprenta, la primera mecanización total de un artesanado, fragmenta el movimiento de la mano en una serie de pasos discretos que son tan repetibles como la rueda es rotatoria. De esta secuencia analítica procede el principio de la línea de montaje, pero la línea de montaje es obsoleta ahora en la época eléctrica porque la sincronización ya no es secuencial. En el caso de las cintas de grabado la sincronización de cualquier número de actos puede ser simultánea. Por tanto, el principio mecánico del análisis en series ha llegado a su fin (p. 152).

La relación entre un medio que floreció en la Grecia clásica, la escritura alfabética, y un medio que alcanzó su desarrollo muchos siglos después, la máquina, resulta extraña sólo si se concentra uno en el uso o contenido de ambos medios, o bien en su naturaleza técnica propiamente dicha, ya que en realidad son muy diferentes. Pero lo importante es que el alfabeto fue la primera forma tecnológica de "mecanización", ya que "[la] mecanización se alcanza mediante la fragmentación de cualquier proceso y la colocación de las partes fragmentarias en una serie (...)" (McLuhan, 1964, p. 11) o secuen$\mathrm{cia}^{15}$. Podríamos decir que sin ser una máquina en sentido material,

15 La escritura alfabética lleva a las palabras escritas y cada palabra escrita supone las letras del alfabeto correspondiente, las cuales forman una secuencia analítica del sonido. McLuhan explica esto en el capítulo 9 de Understanding Media. Para una discusión adicional cfr. Carrillo (2004, 2013). 
en términos de configuración de la experiencia, el alfabeto actúa como una máquina o, si se prefiere, una máquina actúa como el alfabeto: ambos generan una estructura de la experiencia analítica, fragmentadora y secuencial. ${ }^{16}$

Nótese que así como no importa cuál es el producto —el usode la máquina, en términos de la estructura de la experiencia tampoco importa, "ni en lo más mínimo" (McLuhan, 1964, p. 7), si de la escritura resultan biblias, manifiestos comunistas, novelas o textos científicos. Lo que cuenta es la escritura misma, en tanto conformadora de la estructura de la experiencia, no su mensaje o significado discursivo. Lo que importa es todo un hábito mental específico resultante de la escritura alfabética, hábito que se manifiesta en que “(...) la gente altamente alfabetizada tiende a hablar de manera tan conexa y causal como sea posible (...)” (McLuhan, 1964, p. 77), es decir, de acuerdo con secuencias lógicas. Es lo que McLuhan (1964) también llama el "patrón en un solo sentido" (p. 35) —el lineal, de la secuencia-, que equivale a "los viejos patrones de la expansión mecánica, en un solo sentido” (p. 36). En otras palabras, es la escritura alfabética la que genera el set mental que se encuentra realizado en "la industria mecanizada (...) mediante monofractura o al abordar todas las cosas y operaciones una fracción a la vez” (p. 73).

\section{La doble perplejidad ante los medios}

Al haber aclarado la naturaleza de la forma alfabética o mecánica y al tener en cuenta la tesis de McLuhan de que los medios tienen una realidad estructural, queda claro que el método para el estudio de los medios no puede ser el tradicional consistente en una aproximación secuencial a un medio aislado porque los medios tienen sus efectos en una "matriz" de relaciones de los unos con los otros. De hecho, es esa naturaleza estructural o relacional de cada

16 McLuhan (1964) dice que “(...) la mecanización se alcanza mediante la fragmentación de cualquier proceso y mediante la colocación de las partes fragmentadas en una serie." (p. 11) Justo eso es lo que se hace al escribir cada palabra, y de hecho al escribir cada oración al fragmentarla en palabras. 
medio, lo que hace que un medio en sus efectos sociales no pueda ser estudiado en sí mismo. Esto tiene dos consecuencias muy importantes. Una se refiere a la introducción original de un medio en un contexto mediático, en un contexto que, entonces es el, digámoslo así, normal para ese medio, su matriz o entorno mediático originario; en ese entorno originario el medio tiene un efecto total que el hombre alfabético no espera y es incapaz de prever. La otra consecuencia de la realidad estructural del medio es que un medio dado, sacado de su matriz mediática originaria e introducido en otra, ni siquiera tiene los efectos que tuvo en su entorno mediático originario - esto último es el asunto central en los así llamados "problemas del desarrollo"-.

El primer asunto ya lo tratamos, es justo el de la atención al contenido y no al medio mismo en sus efectos. Vimos casos muy claros. Uno de ellos la introducción del aeroplano, el cual tiene un conjunto de usos como meta, eso es lo que el hombre alfabetizado ve desde el principio, desde que concibe el medio y lo diseña, sin embargo, no ve, no prevé su efecto sobre la forma de la ciudad o la política. ${ }^{17}$ En otras palabras, la introducción de un medio tiene dos tipos de efectos, los previstos, buscados, y un conjunto de efectos colaterales que no son previstos. Podríamos decir que esto es lo que McLuhan llama el "efecto total" del medio, la tesis central que subyace a la teoría mediática de McLuhan, en particular a su libro Understanding Media, es que los efectos colaterales son lo que McLuhan (1964) llama la "carga subliminal" (p. 20) que el "análisis de 'contenido" (p. 20) es incapaz de captar. El hecho sería que, según McLuhan, el hombre alfabético o mecánico sufre el efecto total de cada medio "subliminalmente y sin conciencia crítica" (p. 285) ${ }^{18}$. Es por ello que Understanding Media en su conjunto está dedicado a ver los efectos de los medios, se guía en su totalidad por la premisa

17 Recuérdese el texto ya citado antes: “(...) el aeroplano tiende a disolver la forma ferroviaria de la ciudad, la política y la asociación, con total independencia de para qué se usa el aeroplano."

18 El carácter subliminal de la aceptación del efecto total del medio lo desarrolla McLuhan básicamente en el capítulo 4 de Understanding Media bajo el famoso y conocido tema del trance de Narciso. Se trata de un tema especial que, si bien complementa la teoría de McLuhan sobre el método para el estudio de los medios, no es indispensable para el presente texto. 
ya discutida de que "el medio es lo que importa", no su uso o contenido y que el hombre alfabetizado es incapaz de comprender que "lo que importa es el medio".

El segundo aspecto, el trasplante de un medio de un entorno mediático a otro, es uno de los que trata McLuhan en el capítulo 5 del libro que nos ocupa al referirse a las "uniones híbridas" (p. 49) y los "efectos de los híbridos mediáticos" (p. 51) 19. Aquí nos concentramos en señalar la idea básica de que el mismo medio tiene efectos muy diferentes en entornos mediáticos distintos. McLuhan se refiere en especial a tipos de estos casos. Uno muy relevante es el de la introducción de medios provenientes de las sociedades "mecánicas" en las sociedades orales o tribales, en las "culturas atrasadas y no industriales" (p. 27). McLuhan da un ejemplo contundente:

Cuando los misioneros dieron hachas de acero a los nativos australianos, su cultura, basada en el hacha de piedra, colapsó. El hacha de piedra no solamente era escasa sino que siempre había sido un símbolo de estatus básico de la importancia masculina. Los misioneros llevaron grandes cantidades de afiladas hachas de acero y las entregaron a las mujeres y a los niños. Los hombres tenían que conseguirlas prestadas de las mujeres, causando esto el colapso de la dignidad masculina (p. 24).

Es claro que las hachas de piedra no sólo requerían una gran inversión de tiempo y habilidad en su producción, de destreza y fuerza, sino que podemos sospechar que el fabricar un hacha de piedra era el rito iniciático de la masculinidad, un rito esencial para la definición de lo masculino y su habilitación para acceder a las mujeres, uno de los ritos iniciáticos que son tan comunes en las sociedades arcaicas. McLuhan (1964) tipifica este efecto con lo señalado a continuación:

[u]na jerarquía tribal y feudal de la clase tradicional se colapsa rápidamente cuando encuentra cualquier medio (...) del tipo me-

19 Sin embargo, la hibridación no se reduce a dicho trasplante de un entorno a otro. McLuhan (1964) también llama "híbrido" al "encuentro de dos medios” (p. 55), por ejemplo el del cine y el sonido (p. 49). 
cánico, uniforme y repetitivo. El medio de la moneda o la rueda o la escritura, o cualquier otra forma de incremento especialista del intercambio y la información, servirá para fragmentar una estructura tribal (p. 24).

McLuhan nos dio, entonces, un ejemplo muy claro de lo ocurrido en una cultura "atrasada y no industrial", pero en general se trata, según dijimos, de lo que los sociólogos, economistas y politólogos suelen llamar los "problemas del desarrollo" y que tanto ocupan a las agencias internacionales de desarrollo. Se refiere al conjunto de problemas que surgen al intentar modernizar las sociedades prealfabéticas o apenas alfabetizadas, lo cual ha generado toda una montaña de literatura especializada. ${ }^{20}$ Pero en todos los casos se trata de la introducción de algún medio — por ejemplo, el crédito, el banco, el reloj y el automóvil, la universidad, y muchos otros- en una matriz mediática distinta de la original, siendo los resultados - el "efecto total" - muy distintos de los esperados, para tribulación de las agencias internacionales, sus expertos y las capas occidentalizadas de las regiones atrasadas.

Un caso en particular interesante es la introducción de instituciones occidentales en las áreas tribales o "atrasadas". Notorio entre estos casos es el de la democracia. La introducción de esta institución no tiene los mismos efectos, por ejemplo, en México que en Suiza o en Suecia. ${ }^{21}$ Es justo el no captar que los medios tienen una realidad estructural lo que lleva a desatender el efecto que tiene un medio que proviene de un entorno o matriz mediática al insertarlo en otro.

20 Como simple muestra, aunque él todavía no hable en explícito de los "problemas del desarrollo", los varios textos antropológicos de Edward T. Hall, por ejemplo, The Hidden Dimension o bien The Silent Language, son una verdadera mina de casos, problemas y observaciones de lo que ocurre cuando el hombre occidental se confronta con el hombre "oriental", que es otro de los términos que McLuhan utiliza para el hombre tribal o premoderno.

21 Esto, el que la democracia mexicana no funcione como la de, digamos, los países nórdicos, ha llevado a uno de los demócratas más importantes de México, Gabriel Zaid, a una decepción con la democracia. McLuhan diría que no sólo Zaid, sino todos los que se prometieron, aunque no fuera inmediato, un funcionamiento de la democracia mexicana según el modelo nórdico, pensaban y piensan de acuerdo con el modelo secuencial de los alfabetizados. Sobre la decepción de Zaid cfr. Arreola 2017. 
Gran parte de los problemas actuales de algunos países de América Latina - para no hablar de Afganistán o del Cuerno de Áfricaproviene de la introducción de instituciones occidentales en culturas definidas por matrices mediáticas no mecánicas o alfabéticas, es decir, tribales o muy tradicionales, en las que el individualismo occidental nórdico está ausente o muy poco desarrollado.

Los intelectuales y las burocracias locales en las áreas "atrasadas", muchas veces educados en el Occidente, al seguir los modelos alfabéticos de Occidente, van de sorpresa en sorpresa y de decepción en decepción al ver que la tan ansiada modernización mediante la introducción de instituciones y otros medios — "mecánicos” y también "eléctricos" - no produce los resultados que ha tenido en el Occidente propiamente dicho. No entienden qué pasa. Es un problema de "método", de su forma de orientarse en el mundo, que los ciega para la realidad mediática en tanto realidad estructural. De ahí en particular el tópico de la "decepción con la democracia", no sólo en México sino también en otros países de Latino América.

\section{Conclusión: el tweet y el artículo editorial}

Si bien la teoría mediática de McLuhan remite al inicio de la década de los años 50 del siglo xx y la parte medular de la misma se encuentra en los textos The Gutenberg Galaxy (1962) y su continuación como Unterstanding Media. The Extensions of Man (1964), fenómenos como la perplejidad de los analistas alfabéticos ante el triunfo del brexit o el de Trump, en el caso de los países occidentales modelo Reino Unido y Estados Unidos, o bien la perplejidad de los mismos analistas en los países del Tercer Mundo ante los "problemas del desarrollo" o las "modernizaciones inconclusas", sugieren una ruptura entre la realidad mediática y la comprensión de la misma por parte de los analistas. Se puede pensar que si la comprensión de los medios fuera la adecuada, acontecimientos como los señalados no tendrían efectos tan notables, podríamos decir, casi traumáticos, sobre los analistas; estos tendrían que haber sido capaces de prever qué pasaría o por lo menos no habrían sido tomados por sorpresa de manera tan notable.

La disparidad entre los acontecimientos y lo que los analistas esperaban, sugiere, entonces, si McLuhan tiene razón, que en general 
sigue oculto el hecho de que "el medio es lo que importa" — the medium is the message-. Parecería haber una incomprensión acerca del "efecto total" de los medios. Ya en Understanding Media McLuhan señaló cómo el medio de la TV chocaba con ventaja con el del periódico (p. 29), al anticipar de esta manera el triunfo del mediático showman Trump sobre la letrada Clinton y toda la gran prensa que la apoyó sin ambages. Siguiendo a McLuhan podría sugerirse que el triunfo de Trump fue la debacle de los hombres alfabéticos frente a un público "eléctrico" en el entorno digitalizado — la versión más reciente y omniabarcante de los medios "eléctricos"-.

En conclusión, la perplejidad de la mayor parte de los analistas frente a acontecimientos clave de la política contemporánea podría muy bien tener que ver con las relaciones entre medios tales como el editorial de prensa por un lado y el tweet por otro, entre el libro y el análisis de fondo por una parte y la TV y la red, por la otra. Siguiendo a McLuhan podríamos dudar que el editorial de prensa tenga la fuerza que tuvo antaño frente al libro en la época de las redes sociales, dominadas por el tweet, por Facebook y por Instagram, para nombrar sólo a algunas. Si bien el periódico es mucho más rápido que el libro, las redes sociales son todavía más rápidas que el periódico, lo cual puede ser un factor que aclare ciertos eventos políticos de gran alcance y profundidad que ocurren al margen de los contenidos discursivos o, de hecho, a pesar de ellos.

Vale entonces subrayar que en contraste con los analistas, los alfabetizados, McLuhan propuso la "aproximación estructural" a los medios y, ciertamente, como un imperativo que tiene su origen en la realidad misma del entorno mediático "eléctrico", como algo inmanente a él. El paralelismo entre la manera adecuada de estudiar los medios y la realidad mediática misma, es decir, el paralelismo entre el "enfoque estructural" y las "estructuras mediáticas", se expresa como la necesidad inmanente a la realidad mediática, propia de la misma; de aproximarse a ella no secuencial sino estructuralmente. La conclusión es, pues, que la forma de conciencia desplegada por McLuhan y la realidad mediática son conformes o isomórficas, porque tal forma de conciencia surge de la realidad mediática en su fase o "época eléctrica" de manera inmanente. 


\section{Bibliografía}

Arreola, Federico (2017). La decepción democrática de Gabriel Zaid y el asesinato del policía federal en Veracruz. SDP Noticias. 26 de junio de 2017. 06 de agosto de 2017. Disponible en $<$ https:/www.sdpnoticias.com/nacional/2017/06/26/la-decepcion-democratica-de-gabriel-zaid-y-el-asesinato-del-policia-federal-en-veracruz>

Carrillo, Alberto (2004). "La escritura y la estructura de la percepción”. A Parte Rei. Revista electrónica de filosofía. No. 33, 1-5. Disponible en <http://serbal.pntic.mec.es/\%7Ecmunoz11/carrillo33.pdf>

Carrillo, Alberto (2013). Fotografia, cine, juegos digitales y narratividad. Estudios sobre la sensibilidad novomediática. México: BUAP \& Itaca.

Carrillo, Alberto (2017). McLuhan y las estructuras de la experiencia. El caso del alfabeto y el espacio euclidiano. De próxima aparición. Kant, Immanuel (1781). Kritik der reinen Vernunft (Ed. 1990). Hamburg: Felix Meiner Verlag. Disponible en <http://gutenberg.spiegel.de/buch/kritik-der-reinen-vernunft-1-auflage-3508/39>

McLuhan, Marshall (1964). Understanding Media. The Extensions of Man (Ed. 1994). Cambridge: The MIT Prees 